5th International Workshop on Astronomy and

Relativistic Astrophysics (IWARA2011)

International Journal of Modern Physics: Conference Series

Vol. 18 (2012) 25-30

(C) World Scientific Publishing Company

DOI: $10.1142 / S 201019451200815 X$

\title{
CORRECTIONS TO THE FINE STRUCTURE CONSTANT IN (D+1)-DIMENSIONAL GLOBAL MONOPOLE SPACETIME
}

\author{
GEUSA DE A. MARQUES \\ Unidade Acadêmica de Física, Universidade Federal de Campina Grande \\ 58109-790, Campina Grande, Pb, Brazil. \\ gmarques@df.ufcg.edu.br \\ V. B. BEZERRA \\ Departamento de Física, Universidade Federal da Paraiba \\ 58051-970, João Pessoa, Pb, Brazil. \\ valdir@fisica.ufpb.br
}

\begin{abstract}
In this paper we use the Generalized Uncertainty Principle in order to obtain the corrections to the fine structure constant in $(D+1)$-dimensional global monopole spacetime. We also discuss the case $D=3$ corresponding to the $(3+1)$-dimensional global monopole spacetime.
\end{abstract}

Keywords: Global monopole; Fine structure constant; Higher dimensional spacetime.

PACS numbers: 02.40.-k,03.65.Pm

\section{Introduction}

The Standard Cosmological Model preticts that the Universe has experienced a chain of phase transitions with spontaneous symmetry breaking. During this process the so called topological defects arised, as for example, monopoles, strings and domain walls ${ }^{1}$. In particular, global monopoles appear in models with broken global $S O(3)$ symmetry $^{2}$. The model consists of the Higgs triplet of scalar fields with Lagrangian

$$
\mathcal{L}=-\frac{1}{2} \partial_{\mu} \phi^{a} \partial^{\mu} \phi^{a}-\frac{1}{4} \lambda\left(\phi^{a} \phi^{a}-\eta^{2}\right)^{2},
$$

where $a=1,2,3$.

The gravitational field corresponding to a global monopole is represented by the following spherically symmetric line element ${ }^{2}$

$$
d s^{2}=-B(r) d t^{2}+A(r) d r^{2}+r^{2}\left(d \theta^{2}+\sin ^{2} \theta d \varphi^{2}\right)
$$


with the functions $A$ and $B$ given by

$$
B=A^{-1}=1-8 \pi \frac{G \eta^{2}}{c^{4}}-\frac{2 G M(r)}{r c^{2}}
$$

where the parameter $\eta^{2}$ is related with the linear energy density.

The scale of the monopole's core is defined by the Compton wavelength of Higgs bosons, $\varrho \sim \lambda^{-1 / 2} \eta^{-1}$. The parameter $M=\lim _{r \rightarrow \infty} M(r)$ which characterizes the monopole's mass, assumes values in the interval $0 \leq 8 \pi \eta^{2}<1$ and is given, approximately, by ${ }^{3}$

$$
M \approx-\frac{6 \pi \eta}{\sqrt{\lambda}} .
$$

Therefore asymptotically the spacetime of a global monopole corresponds to the Schwarzschild spacetime with negative mass and additional solid angle deficit $32 \pi^{2} \eta^{2}$. The spacetime of point-like monopole is obtained from the above metric by disregarding internal structure of the global monopole but preserving the solid angle deficit. It is described by the following line element

$$
d s^{2}=-c^{2} b_{3}^{2} d t^{2}+\frac{d r^{2}}{b_{3}^{2}}+r^{2}\left(d \theta^{2}+\sin ^{2} \theta d \varphi^{2}\right),
$$

where the parameter $b_{3}^{2}=1-\frac{8 \pi G \eta^{2}}{c^{4}}$, and the subindex in $b$ indicates the dimension of the space. The scalar curvature is given by $R=\frac{1-b_{3}^{2}}{r^{2} b_{3}^{2}}$. Therefore, this spacetime is not flat, but surprisingly there is no Newtonian potential associated to it.

This metric can be written in a different form if we make the following change in time coordinates $t \rightarrow \tau=t / b_{3}$. As a result, it turns into

$$
d s^{2}=-c^{2} d \tau^{2}+\frac{d r^{2}}{b_{3}^{2}}+r^{2}\left(d \theta^{2}+\sin ^{2} \theta d \varphi^{2}\right) .
$$

The main characteristic of this spacetime is the existence of a solid angle deficit $4 \pi\left(1-b_{3}^{2}\right)$. The typical value of the solid angle deficit in the framework of the GUT $\left(\eta^{2} c^{2} L_{p l} \sim 10^{16} \mathrm{GeV}\right)$ is proportional to $1-b_{3}^{2}=\frac{8 \pi G \eta^{2}}{c^{4}} \sim 10^{-5}$.

Taking into account that our proposal is to consider the higher dimensional global monopole spacetime, let us write the generalization of the metric given by eq. (6), for $(D+1)$-dimensional spacetime. In this case, the Euclidean version of the global monopole line element is given by

$$
d s^{2}=d \tau^{2}+\frac{d r^{2}}{b_{D}^{2}}+r^{2} d \Omega_{D-1}^{2},
$$

where $D \geq 3$ and $b_{D}$ is a parameter which codifies the presence of a $(D+1)$ dimensional global monopole. The coordinates are $\left(\tau, r, \theta_{1}, \theta_{2}, \ldots, \theta_{D-2}, \phi\right)$. In this case, the solid angle associated to a hypersphere with unity radius is $\Omega_{b_{D}}=b_{D}^{2} \frac{2 \pi^{D / 2}}{\Gamma\left(\frac{D}{2}\right)}$ which is smaller than the solid angle associated to a hypersphere embbeded in flat spacetime. 


\section{Corrections to the Fine Structure Constant in Higher Dimensions}

In order to obtain the fine structure constant in the $(D+1)$-dimensional global monopole spacetime, let us consider a Hydrogen atom in this background. Thus, the electrostatic force on the electron is given by

$$
\vec{F}_{t o t D}=\left(-\frac{1}{\Omega_{D-1} \epsilon_{D, 0}} \frac{e^{2}}{r^{D-1}}+\frac{S\left(b_{D}\right)}{\Omega_{D-1} \epsilon_{D, 0}} \frac{e^{2}}{r^{D-1}}\right) \hat{r}_{D},
$$

where the first term corresponds to the generalization of the usual Coulomb force to $D$-dimensional space. The second term arises from the geometrical and topological features of the spacetime and corresponds to a generalization of a result obtained in the framework of a $(3+1)$-dimensional global monopole ${ }^{4}$. The factor $S\left(b_{D}\right)$ is also a generalization of previous result ${ }^{4}$ and is given by

$$
S\left(b_{D}\right)=\frac{1}{2} \sum_{n=1}^{\infty} \frac{\left(\pi^{2} \Delta_{D}\right)^{n}}{(n !)^{2}}\left|B_{2 n}\right|\left(1-2^{-2 n}\right)
$$

with $\Delta_{D}=1-b_{D}^{2}$ and $B_{n}$ being the Bernoulli numbers. Thus, we can obtain the Bohr radius in this background, which can be written as

$$
r_{B, D}=\left(\frac{b_{D}^{2} \Omega_{D-1} \epsilon_{D, 0} \hbar^{2}}{m e^{2}}\right)^{\frac{1}{4-D}} \times\left(1-S\left(b_{D}\right)\right)^{4-D}
$$

where $m$ and $e$ are the mass and charge of the electron, respectively. Note that when $D=3$ and $b_{3}=1$, which means absence of global monopole, we obtain the so-called Bohr radius $r_{B, 3}=\frac{4 \pi \epsilon_{0} \hbar^{2}}{m e^{2}}=0.0529 \mathrm{~nm}$ in three dimensional space. This expression for the Bohr radius takes into account that the usual quantization of the angular momentum is now given by $L_{n, D}=\frac{n \hbar}{b_{D}}$.

The fine structure constant in $D$-dimensional space was alreday obtained ${ }^{7}$. It is given by the following expression

$$
\alpha_{D}=\hbar^{2-D} e^{D-1}\left[\Omega_{D-1} \epsilon_{D, 0}\right]^{(1-D) / 2} c^{D-4} G_{D}^{(3-D) / 2}
$$

where $\epsilon_{D, 0}$ is the permittivity constant and $\Omega_{D-1}=\frac{2 \pi^{D / 2}}{\Gamma\left(\frac{D}{2}\right)}$ is the solid angle associated with a hypersphere in $D$ dimensions. From this result, we can obtain a corresponding generalized one in the sense that the global monopole is present. This can be witten as

$$
\hat{\alpha}_{D}=\hbar^{2-D} e^{D-1}\left[b_{D}^{2} \Omega_{D-1} \epsilon_{D, 0}\right]^{(1-D) / 2} c^{D-4} G_{D}^{(3-D) / 2}
$$

where the parameter $b_{D}^{2}$ was introduced to take into account the presence of the $(D+1)$-dimensional global monopole. For $D=3$ and $b_{3}=1$, eq. (12) turns into $\alpha=e^{2} / 4 \pi \epsilon \hbar c \approx 1 / 137$. 
The so-called Generalized Uncertainty Principle ${ }^{5}$ is expected to be satisfied when quantum gravitational effects are important. The interest in this Principle has been motivated by studies in string theory ${ }^{6}$ and gravity ${ }^{5}$. In this context, a minimal length scale is expected to be of the order of Planck length $L_{p}$. This fact leads to corrections to the usual Heisenberg Uncertainty Principle, resulting in the Generalized Uncertainty Principle, which can be expressed as

$$
\Delta x_{i} \Delta p_{i} \geq \hbar\left[1+\beta^{2} L_{p, D}^{2}\left(\frac{\Delta p_{i}}{\hbar}\right)^{2}\right] .
$$

where $\beta$ is a numerical factor of order unity that depends on the specific model and $L_{p, D}$ is the Planck length in $D$ dimensions, which is given by $L_{p, D}=$ $\left(\hbar G_{D} / c^{3}\right)^{1 / D-1}$. Thus, in a three dimensional space eq. (13) implies that the lower bound on the length scale is of the order of Planck's length, $L_{p}=\sqrt{\left(G \hbar / c^{3}\right) \approx}$ $10^{-33} \mathrm{~cm}$, and this plays an important role as a fundamental scale.

Thus, from eq. (13) we can obtain the uncertainty for the momentum which is given by the following relation

$$
\frac{\Delta p_{i}}{\hbar}=\frac{\Delta x_{i}}{2 \beta L_{p, D}^{2}}\left(1-\sqrt{1-\frac{4 \beta^{2} L_{p, D}^{2}}{\Delta x_{i}^{2}}}\right) .
$$

Now, let us consider that the uncertainty in position is equal to the Bohr radius given by eq. (10). Thus, we can write the following relation

$$
\begin{aligned}
\frac{\Delta x_{i}}{L_{p, D}}= & \left(\frac{b_{D}^{2} \Omega_{D-1} \epsilon_{D, 0} M_{p, D}^{3} G_{D}}{m e^{2}}\right)^{\frac{1}{4-D}} \\
& \times\left(1-S\left(b_{D}\right)\right)^{4-D}
\end{aligned}
$$

where $M_{p, D}$ is the Planck mass in $D$ dimensional space, which is given by $M_{p, D}=$ $\left(\hbar^{D-2} / c^{D-4} G_{D}\right)^{1 / D-1}$.

Comparing the Generalized Uncertainty Principle and the Heisenberg Uncertainty Principle, we conclude that the first one can be written formally as

$$
\Delta x_{i} \Delta p_{i} \geq \hbar_{e f f}
$$

where

$$
\hbar_{e f f}=\hbar\left[1+\beta^{2} L_{p, D}^{2}\left(\frac{\Delta p_{i}}{\hbar}\right)^{2}\right] .
$$

Substituting Eqs. (15) into (14) and the result into (16), we get

$$
\begin{gathered}
\hbar\left[1+\frac{1}{4 \beta^{2}}\left(\frac{b_{D}^{2} \Omega_{D-1} \epsilon_{D, 0} M_{p, D}^{3} G_{D}}{m e^{2}}\right)^{\frac{2}{4-D}}\left(1-S\left(b_{D}\right)\right)^{2(4-D)}\right. \\
\left.\hbar_{e f f}=\left(1-\sqrt{1-4 \beta^{2}\left(\frac{m e^{2}}{b_{D}^{2} \Omega_{D-1}\left(1-S\left(b_{D}\right)\right)^{2(4-D)}} \epsilon_{D, 0} M_{p D}^{3} G_{D}\right)^{\frac{2}{4-D}}}\right)^{2}\right] .
\end{gathered}
$$


If we consider a global monopole in the Grand Unified Theory(GUT) framework, the factor $\frac{b_{D}}{\left(1-S_{D}\right)}$ should be approximately equal to unity.

Therefore, the term $\frac{m e^{2}}{\Omega_{D-1} \epsilon_{D, 0} M_{p, D}^{3} G_{D}}$ is much less than one, and therefore can be used as the expansion parameter. Expanding eq. (18) in terms of this quantity, we get

$$
\begin{aligned}
\hbar_{e f f} \simeq & \hbar\left[1+\beta^{2}\left(\frac{m e^{2}}{b_{D}^{2} \Omega_{D-1} \epsilon_{D, 0} M_{p, D}^{3} G_{D}}\right)^{\frac{2}{4-D}}\right. \\
& \left.\times\left(1-S\left(b_{D}\right)\right)^{-2(4-D)}\right] .
\end{aligned}
$$

Note that in the absence of a global monopole, the result given by eq.(18) corresponds to the result obtained by $\mathrm{Nasseri}^{7}$ in $D$-dimensional space. Applying this result given by eq. (18) to $3+1$ dimensional global monopole spacetime, we get

$$
\hbar_{e f f} \simeq \hbar\left(1+9.53 \times 10^{-50} \beta^{2}\right) .
$$

Combining eqs. (12) and (18), we find that the structure constant in a $(D+1)$ dimensional global monopole spacetime is given by the expression

$$
\hat{\alpha}_{D, e f f}=\hbar_{e f f}^{2-D} e^{D-1}\left[b_{D}^{2} \Omega_{D-1} \epsilon_{D, 0}\right]^{(1-D) / 2} c^{D-4} G_{D}^{(3-D) / 2},
$$

which in $3+1$-dimensional global monopole spacetime, turns to

$$
\alpha_{3, \text { eff }}=\frac{e^{2}}{4 \pi \epsilon_{0} \hbar c}\left(1-9.53 \times 10^{-50} \beta^{2}\right) .
$$

\section{Concluding Remarks}

The corrections to the Planck constant and to the fine structure constant are very close to the ones obtained in $D$-dimensional flat spacetime. The difference between $\hbar_{\text {eff }}-\hbar$ in relation to $\hbar$ is of the order of $10^{-49}$. Therefore, the values of the fine structure constant evaluated in the framework of the Generalized Uncertainty Principle and in the presence of a $(D+1)$-dimensional global monopole are very close to the value of the fine structure constant obtained in the framework of the Heisenberg Uncertainty Principle in flat spacetime.

\section{Acknowledgment}

We would like to thank Conselho Nacional de Desenvolvimento Científico e Tecnológico $(\mathrm{CNPq})$ for partial financial support.

\section{References}

1. A. Vilenkin and E. P. S. Shellard, Cosmic string and Other Topological Defects, Cambridge Univ. Press, Cambridge, 1994.

2. Manuel Barriola and A. Vilenkin, Phys. Rev. Lett. 63, 341 (1989).

3. D. Harari and C. Lousto Phys. Rev. D 42, 2626 (1990). 
4. E. R. Bezerra de Mello and C. Furtado, Phys. Rev. D, 56,(1997).

5. M. Maggiore, Phys. Lett. B 304, 65 (1993).

6. D. J. Gross and P. F. Mendle, Nucl. Phys. B 303, 407 (1988); D. J. Gross, Phys. Rev. Lett.60, 1229 (1988); G. Amati, M. Ciafaloni and G. Veneziano, Phys. Lett. B 216, 41 (1989).

7. Forough Nasseri, Phys. Lett. B, 229 (2005). 